\title{
RAPID POLYNOMIAL APPROXIMATION ON STEIN MANIFOLDS
}

\author{
AUĐUNN SKÚTA SNÆBJARNARSON
}

\begin{abstract}
In this paper we generalize to a certain class of Stein manifolds the BernsteinWalsh-Siciak theorem which describes the equivalence between possible holomorphic continuation of a function $f$ defined on a compact set $K$ in $\mathbb{C}^{N}$ to the rapidity of the best uniform approximation of $f$ on $K$ by polynomials. We also generalize Winiarski's theorem which relates the growth rate of an entire function $f$ on $\mathbb{C}^{N}$ to its best uniform approximation by polynomials on a compact set.
\end{abstract}

\section{INTRODUCTION}

The famous Runge-Oka-Weil theorem can be phrased in the following way:

$$
\begin{aligned}
& \text { If } K \subset \mathbb{C}^{N} \text { is compact and polynomially convex, then } \\
& \lim _{n \rightarrow \infty} d_{K}\left(f, \mathcal{P}_{n}\right)=0 \text { for every } f \in \mathcal{O}(K) .
\end{aligned}
$$

Here $\mathcal{P}_{n}$ is the set of polynomials in $\mathbb{C}^{N}$ of degree less than or equal to $n$ and $d_{K}\left(f, \mathcal{P}_{n}\right):=\inf \left\{\|f-p\|_{K}: p \in \mathcal{P}_{n}\right\}$ is the best uniform approximation of $f$ on $K$ by polynomials in $\mathcal{P}_{n}$. In [13] Siciak proves a precise quantitative version of the Oka-Weil-Runge theorem. More specifically, provided certain regularity conditions on $K$, he proves that

$$
\limsup _{n \rightarrow \infty}\left(d_{K}\left(f, \mathcal{P}_{n}\right)\right)^{1 / n} \leq L^{-1}
$$

if and only if $f$ extends as a holomorphic function to $\left\{z \in \mathbb{C}^{N} ; V_{K}(z)<\log (L)\right\}$. Here $V_{K}$ denotes the Siciak-Zakharyuta extremal function defined as the supremum of all entire plurisubharmonic functions $u$ of minimal growth with $\left.u\right|_{K} \leq 0$ (see for example 14 or [1]). Taking Siciak's theorem into consideration, we say that a function $f$ admits to rapid approximation by polynomials on $K$ if (1) is true for some $L>1$. Goncar [8, 9, 10] and Cirka [4, 5] have proven theorems in a similar spirit, regarding rapid approximation by rational functions on $\mathbb{C}^{N}$ (defined in an analogous way).

Following Stoll [16] we say that a manifold $X$ of complex dimension $N$ is $S$-parabolic if it possesses a plurisubharmonic (psh) exhaustion function $\tau$ which is maximal outside a compact subset $S$ of $X$. Such an exhaustion function is called a special 
exhaustion function. The function $\tau$ being maximal outside $S$ is equivalent to $(i \partial \bar{\partial} \tau)^{N}=0$ on $X \backslash S$. We say that an entire function $f$ on $X$ is a $\tau$-polynomial if there are constants $t, C \geq 0$ such that

$$
\log |f(z)| \leq t \tau^{+}(z)+C, \quad z \in X .
$$

In their work [1, 2, 3, Aytuna and Sadullaev consider the Fréchet-space $\mathcal{O}(X)$ of holomorphic functions on $X$. They construct an example of an $S$-parabolic manifold where the $\tau$-polynomials are not dense in $\mathcal{O}(X)$. Zeriahi [19, 20, introduces analogues of classical pluripotential theory to $S$-parabolic Stein manifolds. He generalizes the theorem of Siciak and a theorem of Winiarski [17] to algebraic varieties.

In this paper we consider a Stein manifold $X$ with a psh exhaustion function $\psi$ such that the $(1,1)$-form $\frac{i}{2} \partial \bar{\partial} e^{\psi}$ satisfies certain curvature properties (which we discuss in detail in Section 3). When the curvature properties in question are satisfied, we prove a theorem on rapid approximation by $\psi$-polynomials on compact subsets of $X$. We also prove a generalization of Winiarski's theorem to such manifolds. Our main results are stated in Section 3 and proven in Section 5. In Section 4 we look at a few examples of functions $\psi$ satisfying the aforementioned curvature properties.

Acknowledgments. The author would like to thank his doctoral advisor, Ragnar Sigurðsson, for reviewing this paper and giving helpful comments. This project was funded by The Doctoral Grants of The University of Iceland Research Fund and by The Icelandic Center for Research (Rannís) grant no. 152572-052.

\section{Preliminaries}

Let $X$ be a Stein manifold and $\psi: X \rightarrow \mathbb{R}$ be a plurisubharmonic exhaustion function. The notion of a $\psi$-polynomial on $X$ was introduced by Zeriahi in [19].

Definition 2.1. We say that a function $f \in \mathcal{O}(X)$ is a $\psi$-polynomial if there exist constants $t$ and $C$ such that

$$
\log |f(z)| \leq t \psi^{+}(z)+C, \quad z \in X,
$$

where $\psi^{+}(z):=\max \{0, \psi(z)\}$ is the positive part of $\psi$. We denote by $\mathcal{P}^{\psi}$ the space of $\psi$-polynomials on $X$ and for a fixed $t>0$ we denote by $\mathcal{P}_{t}^{\psi}$ the set of $\psi$-polynomials on $X$ satisfying inequality (2) for some constant $C$. If $f$ is a $\psi$-polynomial on $X$ then the $\psi$-degree of $f$ is

$$
\operatorname{deg}_{\psi}(f):=\inf \left\{t>0: f \in \mathcal{P}_{t}^{\psi}\right\} .
$$

Note that if $X=\mathbb{C}^{N}$ and $\psi(z)=\log \|z\|$ then the notion of a $\psi$-polynomial coincides with the classical notion of a polynomial. The polynomial spaces $\mathcal{P}_{t}^{\tau}$ are of particular interest when the function $\tau$ is a special exhaustion because then they are of finite dimension. More specifically we have: 
Proposition 2.2 ([19], Théorème 4.8). If $\tau$ is a special exhaustion function on the $N$-dimensional manifold $X$, there exists a constant $M$ such that

$$
\operatorname{dim} \mathcal{P}_{n}^{\tau} \leq\left(\begin{array}{c}
N+n M \\
N
\end{array}\right), \quad n \in \mathbb{N} .
$$

In [19, 20, Zeriahi considers the case when $X$ is an affine algebraic variety and proves theorems similar to the theorems of Oka-Weil and Siciak. In [1, 2, 3, Aytuna and Sadullaev consider the polynomial space $\mathcal{P}^{\tau}$ when $\tau$ is a special exhaustion function. They construct an example where the polynomial space $\mathcal{P}^{\tau}$ consist only of the constant functions, and another one where $\mathcal{P}^{\tau}$ is not trivial, but still not dense in the Fréchet-space of holomorphic functions $\mathcal{O}(X)$. As a corollary to our main results of this paper we find sufficient conditions for $\mathcal{P}^{\tau}$ to be dense in $\mathcal{O}(X)$.

As an analogue to the classical Lelong class $\mathcal{L}$ on $\mathbb{C}^{N}$ we define the $\psi$-Lelong class on $X$ to be the set

$$
\mathcal{L}_{\psi}:=\left\{u \in \operatorname{PSH}(X) ; \exists C \geq 0 \text { such that } u \leq \psi^{+}+C \text { on } X\right\}
$$

where $\psi$ is any psh exhaustion on $X$ and we define

$$
\mathcal{L}_{\psi}^{+}:=\left\{u \in \mathcal{L}_{\psi} ; \exists C \geq 0 \text { such that } \psi \leq u^{+}+C \text { on } X\right\} .
$$

If $\psi$ is a special exhaustion function then $\mathcal{L}_{\psi}$ is an abstract Lelong class in the sense of [21]. This means that for any compact non-pluripolar set $K \subset X$ the extremal function

$$
V_{K, \psi}(z):=\sup \left\{v(z) ; v \in \mathcal{L}_{\psi},\left.\quad v\right|_{K} \leq 0\right\}, \quad z \in X
$$

is well defined, i.e. we have $V_{K, \psi}<\infty$. Indeed, the upper semi-continuous regularization $V_{K, \psi}^{*}$ is a member of $\mathcal{L}_{\psi}$. We also define the function

$$
\Phi_{K, \psi}(z)=\sup \left\{|f(z)|^{1 / t} ; f \in \mathcal{P}_{t}^{\psi},\|f\|_{K} \leq 1, t>0\right\} .
$$

In the case when $X=\mathbb{C}^{N}$ and $\psi(z)=\log \|z\|$ the function $\Phi_{K}:=\Phi_{K, \psi}$ was originally introduced by Siciak [13] in order to extend classical results of approximation and interpolation to holomorphic functions of several complex variables. Later, Zakharyuta [18] defined the extremal function $V_{K}:=V_{K, \psi}$ with $X=\mathbb{C}^{N}$ and $\psi=\log \|z\|$. It is well known that $\log \Phi_{K}=V_{K}$ for every compact $K \subset \mathbb{C}^{N}$ (see for example [11, Theorem 5.1.7]), but on a more general manifold such an equality might not be true, even if we assume $\psi$ to be a special exhaustion function. Indeed, as mentioned before, there exists an example of a special exhaustion $\psi$ on a manifold $X$ such that $\mathcal{P}^{\psi}$ consists only of the constants [3], in which case we have $\log \Phi_{K, \psi} \equiv 0$. In general we have $\log \Phi_{K, \psi} \leq V_{K, \psi}$.

\section{RESUlts}

In this section we present the main results of this paper. All results are proven in Section 5. First we must introduce some notation. Recall that if $\omega$ is a Kähler-form on $X$ with coefficients $\omega_{j, \bar{k}}$ with respect to a given coordinate system then the Ricci curvature of $\omega$ is given as follows

$$
\operatorname{Ricci}(\omega)=-i \partial \bar{\partial} \log \left(\operatorname{Det}\left(\omega_{j, \bar{k}}\right)\right) .
$$


For any $z \in X, r>0$ we denote by $B(z, r, \omega)$ the geodesic ball with center $z$ and radius $r$ with respect to the metric $\omega$.

Definition 3.1. Let $\psi$ be a psh exhaustion function on the $N$-dimensional manifold $X$ and assume that the $(1,1)$-form $\frac{i}{2} \partial \bar{\partial} e^{\psi}$ is smooth and strictly positive outside a compact subset $S$ of $X$.

(i) Let $\theta \in \operatorname{PSH}(X)$. We say that $\theta$ is a Ricci compensator for $\psi$ if it is continuous, strictly psh in a neighborhood of $S$,

$$
|\theta| \leq A \psi^{+}+B \quad \text { on } X
$$

for some constants $A, B>0$ and

$$
\frac{i}{2} \partial \bar{\partial} \theta+\operatorname{Ricci}\left(\frac{i}{2} \partial \bar{\partial} e^{\psi}\right) \geq 0, \quad \text { on } X \backslash S .
$$

If there exists a Ricci compensator for $\psi$, then we say that $\psi$ is Ricci compensable.

(ii) We say that $\psi$ induces an integral estimate for holomorphic functions if for every $\delta>0$ there are constants $A, B$ such that for every $z \in X \backslash S$ and every function $F \in \mathcal{O}\left(\bar{B}\left(z, \delta, \frac{i}{2} \partial \bar{\partial} e^{\psi}\right)\right)$

$$
|F(z)|^{2} \leq e^{A \psi^{+}(z)+B} \int_{B\left(z, \delta, \frac{i}{2} \partial \bar{\partial} e^{\psi}\right) \backslash S}|F|^{2}\left(\frac{i}{2} \partial \bar{\partial} e^{\psi}\right)^{N} .
$$

Our first main result is the following.

Theorem 3.2. Let $\psi$ be a psh exhaustion function on $X$ which is Ricci compensable and induces an integral estimate for holomorphic functions. Let $K \subset X$ be compact and $\varphi \in \mathcal{L}_{\psi}^{+}$be a continuous function satisfying $\left.\varphi\right|_{K} \leq 0$. Then for every $\left.L \in\right] 1, \infty[$ and every function $f$ holomorphic on $K_{L}:=\{z \in X ; \varphi(z)<\log (L)\}$ we have

$$
\limsup _{t \rightarrow \infty}\left(d_{K}\left(f, \mathcal{P}_{t}^{\psi}\right)\right)^{1 / t} \leq L^{-1}
$$

Notice that if the function $\psi$ is a member of $\mathcal{L}_{\tau}$ for some special exhaustion function $\tau$ then the polynomial spaces $\mathcal{P}_{t}^{\psi}$ have finite dimension (this follows directly from Proposition 2.2. This means that Theorem 3.2 is, in some sense, the strongest in this case. We should note though, that for a given special exhaustion function $\tau$ it is not always possible to find $\psi \in \mathcal{L}_{\tau}$ satisfying the properties of Definition 3.1 (for instance if $\mathcal{P}^{\tau}$ consists only of constants). We then need larger polynomial spaces if we want to apply Theorem 3.2.

If $\psi \in \mathcal{L}_{\tau}$ for a special exhaustion function $\tau$, then the extremal function $V_{K, \psi}$ is well defined. If $V_{K, \psi}$ happens to be continuous as well, then we can take $\varphi$ to be equal to $V_{K, \psi}$ in Theorem 3.2 . In this case the converse of Theorem 3.2 is true as well:

Proposition 3.3. If $\psi \in \mathcal{L}_{\tau}$ for some special exhaustion function $\tau$ and $f: K \rightarrow \mathbb{C}$ is any function s.t.

$$
\limsup _{t \rightarrow \infty}\left(d_{K}\left(f, \mathcal{P}_{t}^{\psi}\right)\right)^{1 / t} \leq L^{-1}
$$


for some $L>1$, then $f$ is the restriction to $K$ of a function holomorphic on the set $K_{L}=\left\{z \in X ; V_{K, \psi}(z)<\log (L)\right\}$.

Observe that if inequality (3) holds for every $L>0$, then $f$ is the restriction to $K$ of an entire function, also denoted by $f$. If $f$ is of finite order $\varrho$ and of finite type $\sigma$ with respect to $\varrho$, then we have a more precise estimate of $d_{K}\left(f, \mathcal{P}_{t}^{\psi}\right)^{1 / t}$. More precisely, we have a generalization of a theorem of Winiarski [17] for the special case $X=\mathbb{C}^{N}$ and $\psi=\log \|z\|$.

Theorem 3.4. Assume $\psi$ is Ricci compensable and induces an integral estimate for holomorphic function. Further assume that for every $r>0$ there exist constants $A, B$ such that

$$
\int_{\{\psi(z)<\log (L)\}}\left(\frac{i}{2} \partial \bar{\partial} e^{\psi}\right)^{N} \leq e^{A L^{r}+B}, \quad L>1 .
$$

Let $K \subset X$ be compact and $\varphi \in \mathcal{L}_{\psi}^{+}$be a continuous function on $X$ satisfying $\left.\varphi\right|_{K} \leq 0$. Then for any entire function $f$ on $X$ satisfying the growth estimates

(5) $\quad \limsup _{r \rightarrow \infty} \frac{\log ^{+} \log \|f\|_{\{\varphi \leq \log (r)\}}}{\log (r)} \leq \varrho$ and $\limsup _{r \rightarrow \infty} \frac{\log \|f\|_{\{\varphi \leq \log (r)\}}}{r \varrho} \leq \sigma$,

for some $\varrho>0, \sigma \geq 0$, we have

$$
\limsup _{t \rightarrow \infty} t^{1 / \varrho}\left(d_{K}\left(f, \mathcal{P}_{t}^{\psi}\right)\right)^{1 / t} \leq(e \sigma \varrho)^{1 / \varrho} .
$$

If $\psi \in \mathcal{L}_{\tau}$ for some special exhaustion function $\tau$ and we take $\varphi:=V_{K, \psi}$ then the converse holds as well, i.e. if $f$ is a function on $K$ and inequality (6) holds, then $f$ extends to an entire function on $X$ and inequalities (5) are true with $\varphi$ replaced by $V_{K, \psi}$.

Theorems 3.2 and 3.4 are based on a third main result, in which we give an estimate for $d_{K}\left(f, \mathcal{P}_{t}^{\psi}\right)$ for fixed $t$.

Theorem 3.5. Let $\psi$ be a psh exhaustion on $X$ such that $\frac{i}{2} \partial \bar{\partial} e^{\psi}$ is smooth and strictly positive outside a compact set $S$ and assume $\psi$ induces an integral estimate for holomorphic functions. Let $K \subset X$ be compact and $\varphi$ be a continuous psh function on $X$ satisfying:

(i) There is a constant $t_{0}>0$ such that $t_{0} i \partial \bar{\partial} \varphi+\operatorname{Ricci}\left(\frac{i}{2} \partial \bar{\partial} e^{\psi}\right) \geq 0$ on $X \backslash S$ and $i \partial \bar{\partial} \varphi>0$ on $S$,

(ii) $\varphi \in \mathcal{L}_{\psi}$,

(iii) $\left.\varphi\right|_{K} \leq 0$.

Let $L>1, f$ be a function holomorphic on the set $\{z \in X ; \varphi(z)<\log (L)\}$ and $\epsilon \in] 0,(L-1) / 2\left[\right.$. Then there are constants $l, T_{0}$ neither depending on $f$ nor $L$ and a constant $M$ not depending on $f$, such that for any $t \geq T_{0}$ we have

$$
d_{K}\left(f, \mathcal{P}_{t}^{\psi}\right) \leq M\|f\|_{\{\varphi \leq \log (L-\epsilon / 2)\}}\left(\frac{1+\epsilon}{L-\epsilon}\right)^{t-l} .
$$


If $L$ is large enough we can write $M=M_{0}\|\bar{\partial} \chi\|_{L^{2}(X \backslash S)}$ where $M_{0}$ is a constant neither depending on $f$ nor $L$ and $\chi: X \rightarrow \mathbb{R}$ is any $C^{\infty}$ cutoff function with $\chi=1$ on $\{\varphi<\log (L-\epsilon)\}$ and $\chi=0$ on $\{\varphi>\log (L-\epsilon / 2)\}$. Here $\|\bar{\partial} \chi\|_{L^{2}(X \backslash S)}$ denotes the $L^{2}$ norm of $\bar{\partial} \chi$ on $X \backslash S$ with respect to the measure $\left(\frac{i}{2} \partial \bar{\partial} e^{\psi}\right)^{N}$ and the natural norm on $\Lambda^{0,1} T_{X}^{*}$ induced by the metric $\frac{i}{2} \partial \bar{\partial} e^{\psi}$.

\section{EXAMPLES}

We start this section by proving two propositions. We then apply them to construct a few examples of psh exhaustion functions $\psi$ on Stein manifolds which are Ricci compensable and induce an integral estimate for holomorphic functions.

Proposition 4.1. Let $\psi$ be a psh exhaustion on $X$ such that $\frac{i}{2} \partial \bar{\partial} e^{\psi}$ is smooth and strictly positive outside a compact set $S$. If there exist functions $\epsilon, M: X \rightarrow \mathbb{R}_{+}$ and constants $A, B>0$ satisfying

$$
\epsilon(z) \geq e^{-\left(A \psi^{+}(z)+B\right)} \text { and } M(z) \leq e^{A \psi^{+}(z)+B}, \quad z \in X,
$$

such that for every $z \in X \backslash S$, there is a coordinate patch $\xi: B(\zeta, \epsilon(z)) \rightarrow X$ with $\xi(\zeta)=z, \xi^{*}\left(\frac{i}{2} \partial \bar{\partial} e^{\psi}\right) \leq M(z) \omega_{0}$ and $\omega_{0}^{N} \leq M(z)\left(\xi^{*} \frac{i}{2} \partial \bar{\partial} e^{\psi}\right)^{N}$ on $B(\zeta, \epsilon(z))$, then $\psi$ induces an integral estimate for holomorphic functions.

Proof. Let $\delta>0$ and write $r(z)=\delta_{1} \min \left\{\epsilon(z),(M(z))^{-1}\right\}$ where $\delta_{1}=\min \{1, \delta\}$. Since $\xi^{*}\left(\frac{i}{2} \partial \bar{\partial} e^{\psi}\right) \leq M(z) \omega_{0}$ on $B(0, \epsilon(z))$ we have

$$
\xi(B(0, r(z))) \subset B\left(z, \delta_{1}, \frac{i}{2} \partial \bar{\partial} e^{\psi}\right) \subset B\left(z, \delta, \frac{i}{2} \partial \bar{\partial} e^{\psi}\right) .
$$

Let $F$ be a function holomorphic in a neighborhood of the ball $\bar{B}\left(z, \delta, \frac{i}{2} \partial \bar{\partial} e^{\psi}\right)$ and denote by $v_{2 N}$ the volume of the unit ball of dimension $2 N$. Then by the sub-meanvalue inequality on $\mathbb{C}^{N}$ we have

$$
\begin{aligned}
|F(z)|^{2} & =|F \circ \xi(0)|^{2} \leq \frac{1}{v_{2 N}(r(z))^{2 N}} \int_{B(0, r(z))}|F \circ \xi|^{2} \omega_{0}^{N} \\
& \leq \frac{\max \left\{\epsilon^{-1}(z), M(z)\right\}^{2 N}}{v_{2 N} \delta_{1}^{2 N}} \int_{B(0, r(z))}|F \circ \xi|^{2} M(z)\left(\xi^{*} \frac{i}{2} \partial \bar{\partial} e^{\psi}\right)^{N} \\
& \leq \frac{\max \left\{\epsilon^{-1}(z), M(z)\right\}^{2 N} M(z)}{v_{2 N} \delta_{1}^{2 N}} \int_{B\left(z, \delta, \frac{i}{2} \partial \bar{\partial} e^{\psi}\right)} F\left(\frac{i}{2} \partial \bar{\partial} e^{\psi}\right)^{N} .
\end{aligned}
$$

By assumption of the growth rate of $\epsilon$ and $M$ we get the result.

Proposition 4.2. Assume $\psi$ is an exhaustion function of the form

$$
\psi(z)=\log \left(\left|g_{1}(z)\right|^{2}+\ldots+\left|g_{m}(z)\right|^{2}\right), \quad z \in X,
$$

for some holomorphic functions $g_{1}, \ldots, g_{m} \in \mathcal{O}(X)$.

(i) The Ricci curvature of $\frac{i}{2} \partial \bar{\partial} e^{\psi}$ is given with

$$
\operatorname{Ricci}\left(\frac{i}{2} \partial \bar{\partial} e^{\psi}\right)=-i \partial \bar{\partial} \log \left(\sum_{1 \leq j_{1}<\ldots<j_{N} \leq m}\left|\operatorname{Det}\left(\operatorname{Jac}\left(g_{j_{1}}, \ldots, g_{j_{N}}\right)\right)\right|^{2}\right) \text {. }
$$


where the sum is taken over every subcollection $\left\{j_{1} \leq \ldots \leq j_{N}\right\} \subset\{1, \ldots, m\}$ of size $N$ and $\operatorname{Jac}\left(g_{j_{1}}, \ldots, g_{j_{N}}\right)$ is the Jacobian of $g_{j_{1}}, \ldots, g_{j_{N}}$ with respect to any local coordinate system.

(ii) If there exist constants $A, B>0$ such that for every $z \in X$ there exists a subcollection $\left\{g_{j_{1}}, \ldots, g_{j_{N}}\right\} \subset\left\{g_{1}, \ldots, g_{m}\right\}$ and a neighborhood $V$ of $z$ such that $\left(g_{j_{1}}, \ldots, g_{j_{N}}\right)$ maps $V$ bijectively to an open ball in $\mathbb{C}^{N}$ of radius $\epsilon(z) \geq$ $e^{-\left(A \psi^{+}(z)+B\right)}$ and such that for every $g_{k} \in\left\{g_{1}, \ldots, g_{m}\right\} \backslash\left\{g_{j_{1}}, \ldots, g_{j_{N}}\right\}$ we have

$$
i \partial \bar{\partial}\left|g_{k}\right|^{2} \leq e^{A \psi^{+}+B} i \partial \bar{\partial} \sum_{s=1}^{N}\left|g_{j_{s}}\right|^{2} \quad \text { on } V,
$$

then $\psi$ induces an integral estimate for holomorphic functions.

Proof. (i) Let $\left(z_{1}, \ldots, z_{N}\right)$ be some local coordinate chart. We have

$$
i \partial \bar{\partial} e^{\psi}=\sum_{j, k, r} \frac{\partial g_{r}}{\partial z_{j}} \frac{\overline{\partial g_{r}}}{\partial z_{k}} d z_{j} \wedge d \bar{z}_{k}
$$

and therefore, by the Cauchy-Binet formula, we have

$$
\begin{aligned}
\left(i \partial \bar{\partial} e^{\psi}\right)^{N} & =\operatorname{Det}\left(\sum_{r} \frac{\partial g_{r}}{\partial z_{j}} \frac{\overline{\partial g_{r}}}{\partial z_{k}}\right)_{j, k} d V \\
& =\left(\sum_{1 \leq j_{1}<\ldots<j_{N} \leq m}\left|\operatorname{Det}\left(\operatorname{Jac}\left(f_{j_{1}}, \ldots, f_{j_{N}}\right)\right)\right|^{2}\right) d V .
\end{aligned}
$$

The result now follows from definition of the Ricci curvature.

(ii) This is just a special case of Proposition 4.1 where the coordinate patch $\xi$ is defined as the inverse of the map $z \rightarrow\left(g_{j_{1}}(z), \ldots, g_{j_{N}}(z)\right)$.

Now we apply propositions 4.1 and 4.2 to construct a few examples where our main results can be applied.

Polynomials in $\mathbb{C}^{N}$. Let $X=\mathbb{C}^{N}$ and let $g_{1}, \ldots, g_{m}$ be polynomials on $\mathbb{C}^{N}$ s.t.

$$
\psi(z):=\log \left(\left|g_{1}(z)\right|^{2}+\ldots+\left|g_{m}(z)\right|^{2}\right), \quad z \in \mathbb{C}^{N}
$$

is an exhaustion function. Further assume that the Jacobian of the map $z \rightarrow$ $\left(g_{1}(z), \ldots, g_{m}(z)\right)$ has full rank on $\mathbb{C}^{N}$. Then $\psi$ is Ricci compensable and induces an integral estimate for holomorphic functions. We do not prove this here since this is a special case of our next example.

Affine algebraic manifolds. Let $X \subset \mathbb{C}^{M}$ be a non-singular algebraic manifold of dimension $N$ and let $g_{1}, \ldots, g_{m}$ be polynomials on $X$ (i.e. each $g_{j}$ is the restriction of a polynomial on $\mathbb{C}^{M}$ to $X$ ). Assume that the function

$$
\psi(z)=\log \left(\left|g_{1}(z)\right|^{2}+\ldots+\left|g_{m}(z)\right|^{2}\right), \quad z \in X
$$

is an exhaustion function, and further assume that the Jacobian of the map $X \rightarrow$ $\mathbb{C}^{m}, z \rightarrow\left(g_{1}(z), \ldots, g_{m}(z)\right)$ has full rank on $X$. Then $\psi$ is Ricci compensable and 
induces an integral estimate for holomorphic functions. By Rudin [12, after a linear change of variables, we can assume that $X$ is a subset of

$$
\left\{z=\left(z_{1}, \ldots, z_{N}, z_{N+1}, \ldots, z_{M}\right)=\left(z^{\prime}, z^{\prime \prime}\right) \in \mathbb{C}^{M} ;\left\|z^{\prime \prime}\right\| \leq A\left(1+\left\|z^{\prime}\right\|\right)^{B}\right\}
$$

for some positive constants $A, B$. This implies that the function $\tau:=\log \left\|z^{\prime}\right\|$ is a special exhaustion function on $X$. Since $\psi \in \mathcal{L}_{C \tau}$ for $C>0$ large enough, we see that the polynomial spaces $\mathcal{P}_{t}^{\psi}$ have finite dimension.

We now prove that $\psi$ is Ricci compensable and induces an integral estimate for holomorphic functions. Our method is based on Demailly's calculations from the proof of [6, Proposition 10.1]. Indeed, we generalize this result by calculating the Ricci curvature of $\frac{i}{2} \partial \bar{\partial} e^{\psi}$.

Let $P_{1}, \ldots, P_{r}$ be generators of the ideal $I(X)$ of polynomials in $\mathbb{C}^{M}$ vanishing on $X$ and let $s=M-N$ be the codimension of $X$. For each $K=\left\{k_{1}<\ldots<k_{s}\right\} \subset$ $\{1, \ldots, r\}$ and each $L=\left\{l_{1}<\ldots<l_{N}\right\} \subset\{1, \ldots, m\}$ denote by $J_{K, L}$ the determinant of the Jacobian of the functions $g_{l_{1}}, \ldots, g_{l_{N}}, P_{k_{1}}, \ldots, P_{k_{s}}$ on $\mathbb{C}^{M}$. Further write

$$
U_{K}:=X \cap\left\{z \in \mathbb{C}^{M}: d P_{k_{1}} \wedge \ldots \wedge d P_{k_{s}}(z) \neq 0\right\} .
$$

The sets $U_{K}$ form an open cover of $X$ since it is non-singular. Denote by $\left(z_{1}, \ldots, z_{M}\right)$ the standard coordinates on $\mathbb{C}^{M}$, write $T_{0}:=\{N+1, N+2, \ldots, M\}$ and let $w \in U_{K}$ be fixed. Without loss of generality we can assume that $X$ can be parameterized in the variables $\left(z_{1}, \ldots, z_{N}\right)$ in a neighborhood of $w$. That means that in a neighborhood of $w$ in $\mathbb{C}^{M}$ we have

$$
\left|D_{K, T_{0}}\right|^{2}:=\left|\operatorname{Det}\left(\frac{\partial P_{k}}{\partial z_{t}}\right)_{k \in K, t \in T_{0}}\right|^{2} \neq 0
$$

and therefore

$$
\begin{aligned}
& d g_{l_{1}} \wedge d \bar{g}_{l_{1}} \wedge \ldots \wedge d g_{l_{N}} \wedge d \bar{g}_{l_{N}} \wedge d P_{k_{1}} \wedge d \bar{P}_{k_{1}} \wedge \ldots \wedge d P_{k_{s}} \wedge d \bar{P}_{k_{s}} \\
= & \left|J_{K, L}\right|^{2} d z_{1} \wedge d \bar{z}_{1} \wedge \ldots \wedge d z_{M} \wedge d \bar{z}_{M} \\
= & \frac{\left|J_{K, L}\right|^{2}}{\left|D_{K, T_{0}}\right|^{2}} d z_{1} \wedge d \bar{z}_{1} \wedge \ldots \wedge d z_{N} \wedge d \bar{z}_{N} \wedge d P_{k_{1}} \wedge d \bar{P}_{k_{1}} \wedge \ldots \wedge d P_{k_{s}} \wedge d \bar{P}_{k_{s}} .
\end{aligned}
$$

Since the gradients $\nabla P_{k}$ are orthogonal to the tangent space of $X$ we see that when we restrict the forms from equation $\sqrt{9}$ to the submanifold $X$ we have

$$
d g_{l_{1}} \wedge d \bar{g}_{l_{1}} \wedge \ldots \wedge d g_{l_{N}} \wedge d \bar{g}_{l_{N}}=\frac{\left|J_{K, L}\right|^{2}}{\left|D_{K, T_{0}}\right|^{2}} d z_{1} \wedge d \bar{z}_{1} \wedge \ldots \wedge d z_{N} \wedge d \bar{z}_{N} .
$$

Now by applying Proposition $4.2(i)$, and by noticing that the function $\log \left|D_{K, T_{0}}\right|^{2}$ is pluriharmonic in a neighborhood of $w$, we see that

$$
\operatorname{Ricci}\left(\frac{i}{2} \partial \bar{\partial} e^{\psi}\right)=-i \partial \bar{\partial} \log \sum_{|L|=N}\left|J_{K, L}\right|^{2}, \quad \text { on } U_{K} \text {. }
$$

Now let $K_{0}$ be fixed. For any $K \neq K_{0}$ the function

$$
a_{K, K_{0}}:=\log \sum_{|L|=N}\left|J_{K, L}\right|^{2}-\log \sum_{|L|=N}\left|J_{K_{0}, L}\right|^{2}
$$

is pluriharmonic on $U_{K} \cap U_{K_{0}}$ since it is the difference of two local potentials of the Ricci curvature. Moreover, this function is locally bounded from above on $U_{K_{0}}$ 
and since $U_{K_{0}} \backslash U_{K}$ is an analytic subset of $U_{K_{0}}$ the function $a_{K, K_{0}}$ is psh on $U_{K_{0}}$. This is true for all $K$ so the function

$$
\log \sum_{|K|=s} e^{a_{K, K_{0}}}=\log \sum_{|K|=s,|L|=N}\left|J_{K, L}\right|^{2}-\log \sum_{|L|=N}\left|J_{K_{0}, L}\right|^{2}
$$

is plurisubharmonic on $U_{K_{0}}$. Now define the function

$$
\theta=\log \sum_{|K|=s,|L|=N}\left|J_{K, L}\right|^{2}, \quad \text { on } X .
$$

By equations 10 and 11 we see that $i \partial \bar{\partial} \theta+\operatorname{Ricci}\left(\frac{i}{2} \partial \bar{\partial} e^{\psi}\right) \geq 0$ on $X$. Moreover, since the Jacobian of $g_{1}, \ldots, g_{m}$ has full rank on $X$, the functions $\left|J_{K, L}\right|^{2}$ never vanish at the same time on $X$, i.e. we have $\theta>-\infty$ on $X$. By a simple application of Hilbert's Nullstellensatz we can see that there exist constants $A$ and $B$ such that $|\theta| \leq A \psi^{+}+B$ on $X$ and therefore $\theta$ is a Ricci compensator for $\psi$.

By applying Hilbert's Nullstellensatz again we can find constants $A_{1}, B_{1}$ such that for each $z \in X$ we can find $K=\left\{k_{1}, \ldots, k_{s}\right\}$ and $L=\left\{l_{1}, \ldots, l_{N}\right\}$ such that

$$
\left|J_{K, L}(z)\right|^{2} \geq e^{-\left(A_{1} \psi^{+}(z)+B_{1}\right)} \text {. }
$$

Since the derivatives of $\left|J_{K, L}\right|^{2}$ have polynomial growth it is simple to show that $\left(g_{l_{1}}, \ldots, g_{l_{N}}, P_{k_{1}}, \ldots, P_{k_{s}}\right)$ maps a neighborhood of $z$ in $\mathbb{C}^{M}$ bijectively to an open ball in $\mathbb{C}^{M}$ of radius $\epsilon(z) \geq e^{-\left(A_{2} \psi^{+}(z)+B_{2}\right)}$ where $A_{2}, B_{2}$ are constants independent of $z$. Since the functions $P_{k_{1}}, \ldots, P_{k_{s}}$ vanish on $X$ we see that $\left(g_{l_{1}}, \ldots, g_{l_{N}}\right)$ maps a neighborhood of $z$ in $X$ to an open ball in $\mathbb{C}^{N}$ of radius $\epsilon(z)$. Since the functions $g_{j}$ are polynomials it is easy to see that inequality (8) is satisfied for some $A, B$.

The complex torus. Let $X=\mathbb{C}^{N} / \mathbb{Z}^{N}$ be the complex torus and

$$
\psi(z)=\|\operatorname{Im}(z)\|=\|y\|, \quad z=x+i y \in X .
$$

Then $\psi$ is Ricci compensable and induces an integral estimate for holomorphic functions. The function $\psi$ is itself a special exhaustion function so the polynomial spaces $\mathcal{P}_{t}^{\psi}$ have finite dimension. Indeed the functions

$$
\xi_{a}(z):=e^{2 \pi i\langle z, a\rangle}, \quad a \in \mathbb{Z}^{N},\|a\| \leq \frac{t}{2 \pi}, \quad z \in X,
$$

form a basis for $\mathcal{P}_{t}^{\psi}$ for every $t$. In this case we can apply our main theorems to prove classical results from Fourier analysis.

We now prove these statements. It is simple to show that $(i \partial \bar{\partial}\|y\|)^{N}=0$ if $y \neq 0$ so $\psi$ is a special exhaustion function. Now suppose $p \in \mathcal{P}_{t}^{\psi}$ for some $t$. Since $p$ is periodic it is of the form $p(z)=\sum_{a \in \mathbb{Z}^{N}} c_{a} e^{2 \pi i\langle z, a\rangle}$ for some constants $c_{a}$. By the Paley-Wiener theorem we see that $p$ is the Fourier-transform of a distribution with support on $B(0, t) \subset \mathbb{R}^{N}$. Therefore we have $c_{a}=0$ if $\|a\|>\frac{t}{2 \pi}$ and the functions from 12 form a basis for $\mathcal{P}_{t}^{\psi}$.

If $\|y\| \geq 1$, then the largest eigenvalue of the metric

$$
\frac{i}{2} \partial \bar{\partial} e^{\psi}=\frac{i e^{\psi}}{8} \sum_{1 \leq j, k \leq N}\left(\frac{\delta_{j, k}}{\|y\|}+\frac{y_{j} y_{k}(\|y\|-1)}{\|y\|^{3}}\right) d z_{j} \wedge d \bar{z}_{k}
$$


is $\lambda_{1}=e^{\psi} / 4$ and corresponds to the eigenvector $y$. Therefore we can apply Proposition 4.1 with $\epsilon(z)=\frac{1}{2}$ (we can map the torus $X$ to a strip in $\mathbb{C}^{N}$ of width one centered at $z$ ) and $M(z)=\frac{1}{4} e^{\psi(z)+\frac{1}{2}}$ so $\psi$ induces an integral estimate for holomorphic functions. The metric $\frac{i}{2} \partial \bar{\partial} e^{\psi}$ has one more eigenvalue $\lambda_{2}=\frac{e^{\psi}}{4\|y\|}$ which corresponds to the $(N-1)$-dimensional eigenspace of vectors perpendicular to $y$. Therefore we have

$$
\operatorname{Ricci}\left(\frac{i}{2} \partial \bar{\partial} e^{\psi}\right)=-i \partial \bar{\partial} \log \left(\lambda_{1} \lambda_{2}^{N-1}\right)=-N i \partial \bar{\partial} \psi+(N-1) i \partial \bar{\partial} \log \psi
$$

As a Ricci compensator we can take the function

$$
\theta=v+\left\{\begin{array}{lc}
N \psi-(N-1) \log \psi, & \text { if } \psi \geq 2 \\
2 N-(N-1) \log 2, & \text { if } \psi<2
\end{array}\right.
$$

where $v \in \mathcal{L}_{\psi}$ is any function which is strictly psh on $\{\psi<2\}$. It is a simple exercise to show that $\theta$ is indeed psh.

The complement of a graph of a holomorphic function. Let $f: \mathbb{C}^{N-1} \rightarrow \mathbb{C}$ be a holomorphic function in $(N-1)$ variables and define

$$
F(z):=z_{N}-f\left(z^{\prime}\right), \quad z=\left(z^{\prime}, z_{N}\right) \in \mathbb{C}^{N} .
$$

Let $X=\mathbb{C}^{N} \backslash\{F=0\}$ and

$$
\psi(z):=\log \left(\left\|z^{\prime}\right\|^{2}+|F(z)|^{2}+\left|F^{-1}(z)\right|^{2}\right), \quad z \in X .
$$

The function $\psi$ is Ricci compensable and induces an integral estimate for holomorphic functions. In [2, Theorem 4.1] we see that $\tau:=\log \left(\left\|z^{\prime}\right\|+|F(z)-1|^{2}\right)-\log |F(z)|$ is a special exhaustion function. It is easy to see that $\psi \in \mathcal{L}_{C \tau}$ for $C>0$ large enough and therefore the polynomial spaces $\mathcal{P}_{t}^{\psi}$ have finite dimension.

To prove these statements we first observe that applying Proposition $4.2(i)$ gives

$$
\operatorname{Ricci}\left(\frac{i}{2} \partial \bar{\partial} e^{\psi}\right)=-i \partial \bar{\partial} \log \left(1+|F|^{-2}\right)
$$

so we can take $\theta=\log \left(1+|F|^{-2}\right)$ as a Ricci compensator. The map $z \rightarrow\left(z^{\prime}, F^{-1}(z)\right)$ is a bijection from $X$ to $\mathbb{C}^{N-1} \times \mathbb{C}^{*}$ so we can take $\left\{z^{\prime}, F^{-1}\right\}$ as the subcollection mentioned in Proposition 4.2 (ii) and $\epsilon(z)=|F(z)|^{-1}$. We just have to check that (8) from Proposition 4.2 is satisfied. Indeed we have

$$
i \partial \bar{\partial}|F|^{2}=|F|^{4} i \partial \bar{\partial}|F|^{-2} \quad \text { on } X
$$

so $\psi$ induces an integral estimate for holomorphic functions.

\section{Proofs}

In this section we prove all results from Section 3. Recall that $X$ is always a Stein manifold and $\psi$ is a psh exhaustion function on $X$. We start with auxiliary propositions.

Proposition 5.1. Assume that $\frac{i}{2} \partial \bar{\partial} e^{\psi}$ is smooth and strictly positive outside a compact subset $S$ of $X$. Then there exists a Kähler form $\omega$ on $X$ such that $\omega=$ $\frac{i}{2} \partial \bar{\partial} e^{\psi}$ outside a compact subset of $X$. Moreover, the metric $\omega$ is complete. 
Proof. By adding a constant to $\psi$ we can assume that $\left.\psi\right|_{S} \leq-1$. We define

$$
\omega:=\frac{i}{2} \partial \bar{\partial}\left(e^{\Gamma \circ \psi}+\epsilon \chi u\right)
$$

where $\Gamma: \mathbb{R} \rightarrow \mathbb{R}$ is a smooth, increasing and convex function satisfying $\Gamma(x)=$ $-1 / 2$ for $x \leq-1$ and $\Gamma(x)=x$ for $x>0, u$ is any strictly psh function defined in a neighborhood of $\{\psi \leq 0\}, \chi: X \rightarrow[0,1]$ is a smooth function with $\chi=1$ on $\{\psi \leq-1 / 2\}$ and $\chi=0$ on $\{\psi \geq-1 / 4\}$ and $\epsilon$ is a small constant. We clearly have $\omega=\frac{i}{2} \partial \bar{\partial} e^{\psi}$ on $\{\psi>0\}$ and $\omega$ is strictly positive on $\{\psi \leq-1 / 2\} \cup\{\psi \geq-1 / 4\}$. The form $\frac{i}{2} \partial \bar{\partial} e^{\Gamma \circ \psi}$ is strictly positive on $\{-1 / 2 \leq \psi \leq-1 / 4\}$ so by choosing $\epsilon$ small enough we can make $\omega$ strictly positive on $X$.

On $X \backslash\{\psi \leq 0\}$ we have $\omega=\frac{i}{2} e^{\psi}(\partial \psi \wedge \bar{\partial} \psi+\partial \bar{\partial} \psi) \geq \frac{i}{2} \partial \psi \wedge \bar{\partial} \psi$ and therefore $|d \psi|_{\omega}=|\partial \psi+\bar{\partial} \psi|_{\omega} \leq 2|\partial \psi|_{\omega} \leq 2|\partial \psi|_{\partial \psi \wedge} \bar{\partial}=2$. By [7, Lemma VIII 2.4] the metric is complete.

For the rest of the section $\omega$ is a Kähler form as described in Proposition 5.1. By adding a constant to $\psi$ we can always assume that $S=\{\psi \leq 0\}$ and $\omega=\frac{i}{2} \partial \partial e^{\psi}$ on $X \backslash S$. If $v$ is a tangent vector in $X$ we denote by $\omega(v)$ the length of $v$ with respect to the metric $\omega$. Whenever we work in local coordinates $\left(z_{1}, \ldots, z_{N}\right)$ we write

$$
\nabla g=\left(\frac{\partial g}{\partial z_{1}}, \ldots, \frac{\partial g}{\partial z_{N}}\right) \quad \text { and } \quad\langle v, w\rangle=\sum_{j=1}^{N} v_{j} w_{j}
$$

for every function $g \in C^{1}$ and tangent vectors $v, w$.

Lemma 5.2. If $B(z, r, \omega) \cap S=\emptyset$ then $\left|e^{\psi(\zeta) / 2}-e^{\psi(z) / 2}\right| \leq \frac{r}{2}$ for every $\zeta \in$ $B(z, r, \omega)$.

Proof. For every tangent vector $v$ we have

$$
\omega(v)=\frac{i}{2} e^{\psi}(\partial \bar{\partial} \psi+\partial \psi \wedge \bar{\partial} \psi)(v) \geq e^{\psi}|\langle\nabla \psi, v\rangle|^{2}
$$

Let $\gamma:[0,1] \rightarrow X$ be a geodesic from $z$ to $\zeta$ of length $r$. By the fundamental theorem of calculus, and by (13) we have

$$
\begin{aligned}
e^{\psi(\zeta) / 2}-e^{\psi(z) / 2}=\int_{0}^{1} \frac{\partial}{\partial t} e^{(\psi \circ \gamma) / 2} d t & \\
& \leq \frac{1}{2} \int_{0}^{1} e^{(\psi \circ \gamma) / 2}\left|\left\langle\nabla \psi, \gamma^{\prime}\right\rangle\right| d t \leq \frac{1}{2} \int_{\gamma} \omega^{\frac{1}{2}}=\frac{r}{2} .
\end{aligned}
$$

The following theorem is a special case of a famous result by Skoda [15].

Theorem 5.3. Let $\varphi$ be a continuous psh function on $X$ satisfying $\frac{i}{2} \partial \bar{\partial} \varphi+\operatorname{Ricci}(\omega) \geq$ 0 on $X$. Then, for any $(0, q)$-form $f$ with $C^{\infty}$ (resp. $L_{\text {loc }}^{2}$ ) coefficients satisfying $\bar{\partial} f=0$ and $\int_{X}|f|_{\omega}^{2} e^{-\varphi} \omega^{N}<\infty$ there is a $(0, q-1)$-form $u$ with $C^{\infty}$ (resp. $L_{\mathrm{loc}}^{2}$ ) coefficients such that $\bar{\partial} u=f$ and

$$
\int_{X}|u|_{\omega}^{2}\left(e^{\psi}+1\right)^{-2} e^{-2 \varphi} \omega^{N} \leq \frac{1}{2 N} \int_{X} s|f|_{\omega}^{2} e^{-2 \varphi} \omega^{N}
$$


where $s$ is a non-negative function on $X$ which equals 1 on $X \backslash S$.

Proof. First assume that $\varphi$ is $C^{\infty}$. Consider the line bundle $E:=X \times \mathbb{C}$ over $X$ with the trivial projection. On the fibers of $E$ we define the Hermitian product

$$
\left\langle\zeta_{1}, \zeta_{2}\right\rangle_{z}:=\left(1+e^{\psi(z)}\right)^{-2} \zeta_{1} \bar{\zeta}_{2}
$$

and denote by $|\cdot|_{E}^{2}=\left(1+e^{\psi(z)}\right)^{-2}|\cdot|^{2}$ the corresponding norm. Denote by $i \Theta(E)=2 i \partial \bar{\partial} \log \left(1+e^{\psi}\right)$ the Chern curvature tensor on $E$ with respect to this metric (see for example comment (12.6) Chapter V in [7). On $X \backslash S$ we have

$$
i\left(1+e^{\psi}\right)^{2} \Theta(E)=4 \omega+4 e^{2 \psi} i \partial \bar{\partial} \psi \geq 4 \omega
$$

so, by assumption on $\varphi$, we have

$$
i \Theta(E)+\operatorname{Ricci}(\omega)+i \partial \bar{\partial} \varphi \geq \frac{4 \omega}{\left(1+e^{\psi}\right)^{2}}
$$

on $X \backslash S$. Therefore the sum of the eigenvalues of the $(1,1)$-form on the left hand side of (14 with respect to the metric $\omega$ is larger than or equal to $4 N\left(1+e^{\psi}\right)^{-2}$.

Now, if we consider $f$ as a section of the cotangent bundle of $E$, by [7, Theorem VIII 6.5] we can find a $(0, q-1)$ form $u$ such that $\bar{\partial} u=f$ and

$$
\int_{X}|u|_{E}^{2} e^{-2 \varphi} \omega^{N} \leq \frac{1}{4 N} \int_{X} s\left(1+e^{\psi}\right)^{2}|f|_{E}^{2} e^{-2 \varphi} \omega^{N},
$$

where $s$ is a positive function equal to 1 on $X \backslash S$. If we replace $|\cdot|_{E}^{2}$ by $\left(1+e^{\psi(z)}\right)^{-2}|\cdot|^{2}$ the result follows.

If $\varphi$ is not $C^{\infty}$ we get the result by finding a decreasing sequence $\left(\varphi_{n}\right)_{n \in \mathbb{N}}$ of $C^{\infty}$ psh functions such that $\varphi_{n} \searrow \varphi$ in the $L_{\text {loc }}^{1}$ topology and by taking the limit. Since $X$ is Stein, such a sequence exists.

Proof of Theorem 3.5. By assumption $(i)$ and compactness of $S$ we can find a constant $T_{0}$ such that $T_{0} \frac{i}{2} \partial \bar{\partial} \varphi+\operatorname{Ricci}(\omega) \geq 0$. Let $\chi: X \rightarrow \mathbb{R}$ be a $C^{\infty}$ cutoff function with $\chi=1$ on $\{\varphi<\log (L-\epsilon)\}$ and $\chi=0$ on $\{\varphi>\log (L-\epsilon / 2)\}$. For any $t>T_{0}$ we can apply Theorem 5.3 to find a function $u_{t}$ solving the $\bar{\partial}$-equation $\bar{\partial} u_{t}=\bar{\partial}(f \chi)=f \bar{\partial} \chi$ on $X$ and satisfying

$$
\int_{X}\left|u_{t}\right|^{2}\left(e^{\psi}+1\right)^{-2} e^{-2 t \varphi} \omega^{N} \leq \frac{1}{2 N} \int_{X} s|f \bar{\partial} \chi|_{\omega}^{2} e^{-2 t \varphi} \omega^{N} .
$$

Now we define the function $p_{t}:=\chi f-u_{t}$. It is clear that $p_{t}$ is an entire function. Let $z \in X$ be such that $B(z, 1, \omega)$ does not intersect $S$ or the support of $\chi$. Then $u_{t}=p_{t}$ on $B(z, 1, \omega)$.

Since $\psi$ induces an integral estimate for holomorphic functions (Def 3.1 (ii)) we can find constants $A$ and $B$ (not depending on $z$ or $t$ ) such that

$$
\begin{aligned}
& \left|p_{t}(z)\right|^{2} \leq e^{A \psi^{+}(z)+B} \int_{B(z, 1, \omega)}\left|u_{t}\right|^{2} \omega^{N}, \\
& \quad \leq e^{A \psi^{+}(z)+B} \sup _{\zeta \in B(z, 1, \omega)}\left(e^{2 t \varphi(\zeta)}\left(e^{\psi(\zeta)}+1\right)^{2}\right) \int_{X}\left|u_{t}\right|^{2}\left(e^{\psi}+1\right)^{-2} e^{-2 t \varphi} \omega^{N} .
\end{aligned}
$$


Since $\varphi \in \mathcal{L}_{\psi}$ we can now use inequality 15 and Lemma 5.2 to show that $p_{t} \in \mathcal{P}_{l+t}^{\psi}$ where $l=\frac{A}{2}+1$.

Let $r>0$ be small enough such that $B(z, r, \omega) \subset \subset\{\varphi<\log (1+\epsilon)\} \subset\{\chi=1\}$ for all $z \in K$. Then $u_{t} \in \mathcal{O}(\bar{B}(z, r, \omega))$ for $z \in K$. Since $\psi$ induces an integral estimate for holomorphic functions, and by the compactness of the sets $S$ and $K$ we can find a positive constant $C$ such that for every $z \in K$ we have

$$
\begin{aligned}
\left|f(z)-p_{t}(z)\right|^{2} & =\left|u_{t}(z)\right|^{2} \leq C\left(\sup _{\zeta \in B(z, r, \omega)} 1+e^{\psi(\zeta)}\right)^{-2} \int_{B(z, r, \omega)}\left|u_{t}\right|^{2} \omega^{N} \\
& \leq C\left(\sup _{\zeta \in B(z, r, \omega)} e^{2 t \varphi(\zeta)}\right) \int_{X}\left|u_{t}\right|^{2}\left(e^{\psi}+1\right)^{-2} e^{-2 t \varphi} \omega^{N} .
\end{aligned}
$$

By the definition of $r$ we can estimate $\sup _{\zeta \in B(z, r, \omega)} e^{2 t \varphi(\zeta)}$ with $(1+\epsilon)^{2 t}$ and recall that we have $\log (L-\epsilon / 2)>\varphi>\log (L-\epsilon)$ on the support of $\bar{\partial} \chi$. By inequalities (15) and (16) we therefore have

$$
\begin{aligned}
\left|f(z)-p_{t}(z)\right|^{2} & \leq C(1+\epsilon)^{2 t} \int_{X} s|f \bar{\partial} \chi|_{\omega}^{2} e^{-2 t \varphi} \omega^{N} \\
& \leq C\|s\|_{X}\left(\frac{1+\epsilon}{L-\epsilon}\right)^{2 t}\|f\|_{\{\varphi<\log (L-\epsilon / 2)\}}^{2}\|\bar{\partial} \chi\|_{L^{2}(X)}^{2}, \quad z \in K
\end{aligned}
$$

where $\|s\|_{X}$ is the sup-norm of $s$ on $X$ and $\|\bar{\partial} \chi\|_{L^{2}(X)}^{2}$ is the $L^{2}$ norm of $\bar{\partial} \chi$ with respect to the metric $\omega$ and measure $\omega^{N}$. We have already seen that $p_{t} \in \mathcal{P}_{l+t}^{\psi}$ and by replacing $t$ with $t-l$ we have the result with $M=C\|s\|_{X}\|\bar{\partial} \chi\|_{L^{2}(X)}$. If $L$ is large enough the support of $\bar{\partial} \chi$ does not intersect $S$ and $\|\bar{\partial} \chi\|_{L^{2}(X)}=\|\bar{\partial} \chi\|_{L^{2}(X \backslash S)}$.

Lemma 5.4. Let $L, \epsilon>0, \varphi \in \mathcal{L}_{\psi}^{+}$be continuous and $\theta$ be a continuous function satisfying the growth condition

$$
|\theta(z)| \leq A \psi^{+}(z)+B, \quad z \in X
$$

for some constants $A, B$. Write $\tilde{\varphi}_{t}:=\left(1-t^{-1}\right) \varphi+t^{-1} \theta$. Then there exists $T$ such that for $t>T$ we have

$$
\left\{z \in X ; \tilde{\varphi}_{t}(z)<L-\epsilon\right\} \subset\{z \in X ; \varphi(z)<L\},
$$

and the function $\tilde{\varphi}_{t}$ is an exhaustion function.

Proof. It is trivial to check that

$$
\{z \in X ; \theta(z)-\varphi(z) \geq 0\} \cap\left\{z \in X ; \tilde{\varphi}_{t}(z)<L-\epsilon\right\} \subset\{z \in X ; \varphi(z)<L\}
$$

for every $t>0$. Therefore we only need to show that

$$
\{z \in X ; \theta(z)-\varphi(z)<0\} \cap\left\{z \in X ; \tilde{\varphi}_{t}(z)<L-\epsilon\right\} \subset\{z \in X ; \varphi(z)<L\}
$$

for large enough $t$.

Since $\varphi \in \mathcal{L}_{\psi}^{+}$and by 17 we can find positive constants $C_{1}$ and $C_{2}$ such that

$$
|\theta(z)| \leq C_{1} \varphi^{+}(z)+C_{2}, \quad z \in X .
$$


If $t>2$ and $z$ is such that $\varphi(z)>1$, then we have

$$
\begin{aligned}
\tilde{\varphi}_{t}(z)=\left(1-t^{-1}\right) \varphi(z)+t^{-1} \theta(z) & >\frac{1}{2}\left(\varphi(z)-\frac{2}{t}|\theta(z)|\right) \\
& \geq \frac{\varphi(z)}{2}\left(1-\frac{2 C_{1}}{t}\right)-\frac{C_{2}}{t} .
\end{aligned}
$$

Since $\varphi$ is an exhaustion, inequality (18) implies that $\tilde{\varphi}_{t}$ is also an exhaustion for $t \geq T_{0}:=\max \left\{2 C_{1}+1,2\right\}$. In particular the set $\left\{\tilde{\varphi}_{T_{0}} \leq L-\epsilon\right\}$ is compact so the continuous function $\theta-\varphi$ has a lower bound $-M<0$ on it. We define $T:=\max \left\{T_{0}, \frac{M}{\epsilon}\right\}$. Now let $t>T$ and $z \in\{\theta-\varphi<0\} \cap\left\{\tilde{\varphi}_{t}<L-\epsilon\right\}$. Since $\theta(z)-\varphi(z)<0$ and $t>T_{0}$ we have $\tilde{\varphi}_{T_{0}}(z)<\tilde{\varphi}_{t}(z) \leq L-\epsilon$ so $\theta(z)-\varphi(z) \geq-M$ and

$$
\varphi(z)=\tilde{\varphi}_{t}(z)-t^{-1}(\theta(z)-\varphi(z))<L-\epsilon-\frac{\epsilon}{M}(-M)=L .
$$

Proof of Theorem 3.2. Let $\epsilon \in] 0,(L-1) / 2[$ and let $\theta$ be a Ricci compensator for $\psi$. By adding a constant to $\theta$ we can assume $\theta<0$ on $K$. Now apply Lemma 5.4 to find $T>0$ such that $\left\{\tilde{\varphi}_{T}<\log (L-\epsilon)\right\} \subset\{\varphi<\log (L)\}$ where $\tilde{\varphi}_{T}:=\left(1-T^{-1}\right) \varphi+T^{-1} \theta$. We can now apply Theorem 3.5 with $\varphi$ replaced by $\tilde{\varphi}_{T}$ and with $L$ replaced by $L-\epsilon$. For $t$ large enough we have

$$
d_{K}\left(f, \mathcal{P}_{t}^{\psi}\right) \leq M\|f\|_{\left\{\tilde{\varphi}_{T} \leq \log (L-3 \epsilon / 2)\right\}}\left(\frac{1+\epsilon}{L-2 \epsilon}\right)^{t-l} .
$$

and

$$
\limsup _{t \rightarrow \infty}\left(d_{K}\left(f, \mathcal{P}_{t}^{\psi}\right)\right)^{1 / t} \leq \limsup _{t \rightarrow \infty}\left(\frac{1+\epsilon}{L-2 \epsilon}\right)^{\frac{t-l}{t}}=\frac{1+\epsilon}{L-2 \epsilon} .
$$

Since $\epsilon>0$ was arbitrary the result follows.

Proof of Proposition 3.3. By assumption there is a sequence $\left(p_{n}\right)_{n \in \mathbb{N}}$ of functions on $X$ such that $p_{n} \in \mathcal{P}_{n}^{\psi}$ for all $n \in \mathbb{N}$ and $\left\|p_{n}-f\right\|_{K} \leq(L-\epsilon(n))^{-n}$ where $\epsilon: \mathbb{N} \rightarrow \mathbb{R}_{+}$is a decreasing function satisfying $\lim _{n \rightarrow \infty} \epsilon(n)=0$. We claim that the sum $p_{1}+\sum_{n=1}^{\infty}\left(p_{n+1}-p_{n}\right)$ is uniformly convergent on compact subsets of $\left\{V_{K, \psi}<\log (L)\right\}$. Indeed for $l<L$ we have

$$
\begin{aligned}
& \left\|p_{n}(z)-p_{n-1}(z)\right\|_{\left\{V_{K, \psi}(z) \leq l\right\}} \leq\left\|p_{n}-p_{n-1}\right\|_{K}\left\|e^{V_{K, \psi}}\right\|_{\left\{V_{K, \psi}(z) \leq l\right\}} \\
& \leq\left(\left\|p_{n}-f\right\|_{K}+\left\|f-p_{n-1}\right\|_{K}\right) l^{n} \leq 2 l\left(\frac{l}{L-\epsilon(n-1)}\right)^{n-1} .
\end{aligned}
$$

Since $l<L$ and $\epsilon(n)$ converges to 0 the series converges. It is obviously equal to $f$ on $K$.

Lemma 5.5. For $a, \varrho>0$ we have

$$
\sum_{n=1}^{\infty}\left(\frac{a}{n}\right)^{n / \varrho} \leq 1+2^{\varrho} a e^{\frac{a}{e \varrho}} .
$$


Proof. Since $(a / n)^{n / \varrho}<2^{-n}$ for $n>\left\lfloor 2^{\varrho} a\right\rfloor$ we have $\sum_{n=\left\lfloor 2^{\varrho} a\right\rfloor+1}^{\infty}\left(\frac{a}{n}\right)^{n / \varrho} \leq 1$. The function $x \rightarrow(a / x)^{x / \varrho}$ is maximized when $x=a / e$. Therefore

$$
\sum_{n=1}^{\left\lfloor 2^{\varrho} a\right\rfloor}\left(\frac{a}{n}\right)^{n / \varrho} \leq\left\lfloor 2^{\varrho} a\right\rfloor\left(\frac{a}{a / e}\right)^{\frac{a / e}{\varrho}} \leq 2^{\varrho} a e^{\frac{a}{e \varrho}} .
$$

Lemma 5.6. Let $\varphi \in \mathcal{L}_{\psi}^{+}$and $L_{0}>1$ be large enough such that $\{\psi<1\} \subset\{\varphi<$ $\left.\log \left(L_{0}\right)\right\}$. Then for any constants $L_{1}, L_{2}$ with $L_{2}>L_{1}>L_{0}$ there exists a function $\chi \in C^{\infty}(X)$ with $\chi=1$ on $\left\{\varphi<\log \left(L_{1}\right)\right\}, \chi=0$ on $\left\{\varphi>\log \left(L_{2}\right)\right\}$ and $\|\bar{\partial} \chi\|_{L^{2}(X)}^{2} \leq \frac{M_{1} L_{2}^{2}}{L_{2}-L_{1}} \int_{\left\{\psi \leq \log \left(L_{2}\right)+M_{2}\right\}} \omega^{N}$ where $M_{1}$ and $M_{2}$ are constants independent of $L_{1}$ and $L_{2}$.

Proof. Let $\chi_{0} \in C^{\infty}(\mathbb{R})$ be such that $\chi_{0}(x)=1$ if $x \leq 1$ and $\chi_{0}(x)=0$ if $x \geq L_{2} / L_{1}$. We can choose such a function such that $\left\|\chi_{0}^{\prime}\right\|_{\mathbb{R}} \leq 2\left(L_{2} / L_{1}-1\right)^{-1}$. Now define

$$
\chi(z)=\chi_{0}\left(e^{\varphi(z)} / L_{1}\right), \quad z \in X .
$$

We clearly have $\chi=1$ on $\left\{\varphi<\log \left(L_{1}\right)\right\}$ and $\chi=0$ on $\left\{\varphi>\log \left(L_{2}\right)\right\}$ so we only have to prove the estimate for $\|\bar{\partial} \chi\|_{L^{2}(X)}^{2}$. First notice that

$$
\begin{aligned}
|\bar{\partial} \chi|_{\omega}^{2} \omega^{N} & \leq \frac{\left\|\chi_{0}^{\prime}\right\|_{\mathbb{R}}}{L_{1}}\left|\bar{\partial} e^{\varphi}\right|_{\omega}^{2} \omega^{N} \leq \frac{2}{L_{2}-L_{1}}\left|\bar{\partial} e^{\varphi}\right|_{\omega}^{2} \omega^{N} \\
& =\frac{2 i}{L_{2}-L_{1}} \partial e^{\varphi} \wedge \bar{\partial} e^{\varphi} \wedge \omega^{N-1} \leq \frac{i}{L_{2}-L_{1}}\left(\partial \bar{\partial} e^{2 \varphi}\right) \wedge \omega^{N-1} .
\end{aligned}
$$

By assumption there exists a constant $C$ such that $\varphi^{+}-C \leq \psi^{+} \leq \varphi^{+}+C$ on $X$. Let $\Gamma_{0} \in C^{\infty}(\mathbb{R})$ be such that $\Gamma_{0}(x)=1$ if $1 \leq x \leq \log \left(L_{2}\right)+C$ and $\Gamma_{0}=0$ if $x \leq 0$ or $x \geq \log \left(L_{2}\right)+C+1$. The function $\Gamma_{0}$ can be chosen such that $\max \left\{\left\|\Gamma_{0}^{\prime}\right\|_{\mathbb{R}},\left\|\Gamma_{0}^{\prime \prime}\right\|_{\mathbb{R}}\right\} \leq 4$. Now define $\Gamma:=\Gamma_{0} \circ \psi$. Then $\Gamma$ equals 1 on the support of the $(0,1)$-form $\bar{\partial} \chi$ and the support of $\Gamma$ is a subset of

$$
F:=\left\{0 \leq \psi(z) \leq \log \left(L_{2}\right)+C+1\right\} \subset X .
$$

Recall that we have $\omega=\frac{i}{2} \partial \bar{\partial} e^{\psi}$ on $F$. By $\sqrt{19}$ we have

$$
\begin{aligned}
\|\bar{\partial} \chi\|_{L^{2}(X)}^{2} & =\int_{X} \Gamma|\bar{\partial} \chi|_{\omega}^{2} \omega^{N} \leq \frac{1}{L_{2}-L_{1}} \int_{F} \Gamma\left(i \partial \bar{\partial} e^{2 \varphi}\right) \wedge \omega^{N-1} \\
& =\frac{1}{L_{2}-L_{1}} \int_{F} e^{2 \varphi}(i \partial \bar{\partial} \Gamma) \wedge \omega^{N-1} \\
& \leq \frac{1}{L_{2}-L_{1}} \int_{F} 4 e^{2(\psi+C)} i(\partial \psi \wedge \bar{\partial} \psi+\partial \partial \bar{\psi}) \wedge \omega^{N-1} \\
& \leq \frac{8 L_{2}^{2} e^{2 C+1}}{L_{2}-L_{1}} \int_{F} e^{-\psi} \omega^{N} \leq \frac{8 L_{2}^{2} e^{2 C+1}}{L_{2}-L_{1}} \int_{\left\{\psi \leq \log \left(L_{2}\right)+C+1\right\}} \omega^{N} .
\end{aligned}
$$

Proof of Theorem 3.4. Assume inequalities (5) are true and let $\epsilon>0$. We first consider the case when $\varphi$ satisfies $(i)$ from Theorem 3.5. By assumption there is a 
constant $C$ such that

$$
\|f\|_{\{\varphi \leq \log (r)\}} \leq C \exp \left((\sigma+\epsilon) r^{\varrho}\right), \quad r \geq 1 .
$$

Since $f$ is entire, inequality (7) from Theorem 3.5 is true for all $L>1$ and all large $t$. In particular if we take $L=L(t):=(t /(\varrho \sigma))^{\varrho^{-1}}+\epsilon / 2$ then by 7 and 20 we have

$$
d_{K}\left(f, \mathcal{P}_{t}^{\psi}\right) \leq M_{0} C \exp \left(\frac{(\sigma+\epsilon) t}{\varrho \sigma}\right)\left\|\bar{\partial} \chi_{t}\right\|_{L^{2}(X)}\left(\frac{1+\epsilon}{L(t)-\epsilon}\right)^{t-l}
$$

for every $t$ large enough. Here $\chi_{t}$ is a cut-off function with $\chi_{t}=1$ on $\{\varphi<$ $\log (L(t)-\epsilon)\}$ and $\chi_{t}=0$ on $\{\varphi>\log (L(t)-\epsilon / 2)\}$. By Lemma 5.6 and assumption (4) (taking $r=\varrho / 2$ ) there are constants $M_{1}, M_{2}, A, B$ such that

$$
\begin{aligned}
\left\|\bar{\partial} \chi_{t}\right\|_{L^{2}(X)}^{2} & \leq \frac{M_{1}(L(t)-\epsilon / 2)^{2}}{\epsilon / 2} \int_{\left\{\psi \leq \log (L(t)-\epsilon / 2)+M_{2}\right\}} \omega^{N} \\
& \leq \frac{M_{1}(L(t)-\epsilon / 2)^{2}}{\epsilon / 2} \exp \left(A e^{\frac{M_{2} \varrho}{2}}(L(t)-\epsilon / 2)^{\frac{\varrho}{2}}+B\right) \\
& =\frac{2 M_{1}}{\epsilon}\left(\frac{t}{\varrho \sigma}\right)^{2 \varrho^{-1}} \exp \left(A e^{\frac{M_{2} \varrho}{2}}\left(\frac{t}{\varrho \sigma}\right)^{\frac{1}{2}}+B\right) .
\end{aligned}
$$

Now, combining (21) and 22 , we get

$$
\limsup _{t \rightarrow \infty} t\left(d_{K}\left(f, \mathcal{P}_{t}^{\psi}\right)\right)^{\varrho / t} \leq \exp \left(\frac{\sigma+\epsilon}{\sigma}\right)(1+\epsilon)^{\varrho} \varrho \sigma .
$$

Since (23) is true for all $\epsilon>0$ the result follows.

Now consider the case when $\varphi$ does not satisfy $(i)$ from Theorem 3.5. Let $\theta$ be a Ricci compensator for $\psi$ satisfying $\left.\theta\right|_{K} \leq 0$, and let $T$ be large enough such that $\tilde{\varphi}_{T}$ (as defined in Lemma 5.4 is an exhaustion function. Then clearly the function $\varphi_{\epsilon}:=(1-\epsilon) \varphi+\epsilon \tilde{\varphi}_{T}$ satisfies $(i)-($ iii $)$ from Theorem 3.5. Moreover, since $\tilde{\varphi}_{T}$ is an exhaustion, we have

$$
\text { and } \begin{aligned}
\left\{\varphi_{\epsilon} \leq \log (r)\right\} & \subset\{(1-\epsilon) \varphi \leq \log (r)\}, \\
\text { af } \quad\|f\|_{\left\{\varphi_{\epsilon} \leq \log (r)\right\}} & \leq\|f\|_{\left\{\varphi \leq \log \left(r^{1 /(1-\epsilon)}\right)\right\}}
\end{aligned}
$$

for $r>1$ large enough. By assumption (5) and by (24) we have

$$
\limsup _{r \rightarrow \infty} \frac{\log ^{+} \log \|f\|_{\left\{\varphi_{\epsilon} \leq \log (r)\right\}}}{\log (r)} \leq \limsup _{r \rightarrow \infty} \frac{\log ^{+} \log \|f\|_{\left\{\varphi \leq \log \left(r^{1 /(1-\epsilon)}\right)\right\}}}{(1-\epsilon) \log \left(r^{1 /(1-\epsilon)}\right)} \leq \frac{\varrho}{1-\epsilon}
$$

and

$$
\limsup _{r \rightarrow \infty} \frac{\log \|f\|_{\left\{\varphi_{\epsilon} \leq \log (r)\right\}}}{r^{\varrho /(1-\epsilon)}} \leq \limsup _{r \rightarrow \infty} \frac{\log \|f\|_{\left\{\varphi \leq \log \left(r^{1 /(1-\epsilon)}\right)\right\}}}{\left(r^{1 /(1-\epsilon)}\right)^{\varrho}} \leq \sigma .
$$

We can now apply our previous conclusion with $\varphi$ replaced by $\varphi_{\epsilon}$ and $\varrho$ replaced by $\varrho /(1-\epsilon)$ and we have

$$
\limsup _{t \rightarrow \infty} t\left(d_{K}\left(f, \mathcal{P}_{t}^{\psi}\right)\right)^{\varrho /((1-\epsilon) t)} \leq e \frac{\varrho}{1-\epsilon} \sigma .
$$

Since this is true for every $\epsilon>0$ the result follows. 
To prove the converse, assume inequality $(6)$ is true with $\varphi$ replaced by $V_{K, \psi}$. Then for $\epsilon>0$ we can find $M$ such that for any $n \geq M$ there is a function $p_{n} \in \mathcal{P}_{n}^{\psi}$ such that

$$
\left\|f-p_{n}\right\|_{K} \leq\left(\frac{e \varrho \sigma(1+\epsilon)}{n}\right)^{n / \varrho} .
$$

Consider the function $G:=p_{M}+\sum_{n=M}^{\infty}\left(p_{n+1}-p_{n}\right)$. Clearly we have $G=f$ on $K$. Moreover, by 25 and Lemma 5.5, we have

$$
\begin{aligned}
|G| & \leq\left|p_{M}\right|+\sum_{n=M}^{\infty}\left|p_{n+1}-p_{n}\right| \leq\left\|p_{M}\right\|_{K} e^{M V_{K, \psi}}+\sum_{n=M}^{\infty}\left\|p_{n+1}-p_{n}\right\|_{K} e^{(n+1) V_{K, \psi}} \\
& \leq\left\|p_{M}\right\|_{K} e^{M V_{K, \psi}}+\sum_{n=M}^{\infty}\left(\left\|p_{n+1}-f\right\|_{K}+\left\|p_{n}-f\right\|_{K}\right) e^{(n+1) V_{K, \psi}} \\
& \leq\left\|p_{M}\right\|_{K} e^{M V_{K, \psi}}+2 e^{V_{K, \psi}} \sum_{n=M}^{\infty}\left(\frac{e \varrho \sigma(1+\epsilon) e^{\varrho V_{K, \psi}}}{n}\right)^{n / \varrho} \\
& \leq\left\|p_{M}\right\|_{K} e^{M V_{K, \psi}}+2 e^{V_{K, \psi}}\left(1+2^{\varrho} e \varrho \sigma(1+\epsilon) \exp \left(\varrho V_{K, \psi}+\sigma(1+\epsilon) e^{\varrho V_{K, \psi}}\right)\right) .
\end{aligned}
$$

In particular we have

$$
\|G\|_{\left\{V_{K, \psi} \leq \log (r)\right\}} \leq\left\|p_{M}\right\|_{K} r^{M}+2 r\left(1+2^{\varrho} e \varrho \sigma(1+\epsilon) r^{\varrho} e^{\sigma(1+\epsilon) r^{\varrho}}\right)
$$

and now it is easy to see that

$$
\limsup _{r \rightarrow \infty} \frac{\log \|G\|_{\{\varphi \leq \log (r)\}}}{r^{\varrho}} \leq \sigma(1+\epsilon)
$$

and the second inequality of (5) follows by letting $\epsilon \rightarrow 0$. By simple calculus we can show that the first inequality of $(5)$ follows from the second one.

\section{REFERENCES}

[1] A. Aytuna and A. Sadullaev. S*-parabolic manifolds. TWMS J. Pure Appl. Math., 2(1):6-9, 2011.

[2] A. Aytuna and A. Sadullaev. Parabolic Stein manifolds. Math. Scand., 114(1):86-109, 2014.

[3] Aydı n Aytuna and Azimbay Sadullaev. Polynomials on parabolic manifolds. In Topics in several complex variables, volume 662 of Contemp. Math., pages 1-22. Amer. Math. Soc., Providence, RI, 2016.

[4] E. M. Čirka. Meromorphic continuation, and the rate of rational approximations in $C^{N}$. Mat. Sb. (N.S.), 99(141)(4):615-625, 1976.

[5] E. M. Črka. Rational approximations of holomorphic functions with singularities of finite order. Mat. Sb. (N.S.), 100(142)(1):137-155, 166, 1976.

[6] Jean-Pierre Demailly. Mesures de Monge-Ampère et caractérisation géométrique des variétés algébriques affines. Mém. Soc. Math. France (N.S.), (19):124, 1985. 
[7] J.P. Demailly. Complex analytic and differential geometry, (version of thursday june 21, 2012). Free accessible book (https://www-fourier.ujf-grenoble.fr/ demailly/documents.html), retrieved 17.11.2015.

[8] A. A. Gončar. Local conditions for the single-valuedness of analytic functions. Mat. Sb. (N.S.), 89(131):148-164, 167, 1972.

[9] A. A. Gončar. A local condition for the single-valuedness of analytic functions of several variables. Mat. Sb. (N.S.), 93(135):296-313, 327, 1974.

[10] A. A. Gončar. On a theorem of Saff. Mat. Sb. (N.S.), 94(136):152-157, 160, 1975.

[11] Maciej Klimek. Pluripotential theory, volume 6 of London Mathematical Society Monographs. New Series. The Clarendon Press, Oxford University Press, New York, 1991. Oxford Science Publications.

[12] Walter Rudin. A geometric criterion for algebraic varieties. J. Math. Mech., 17:671-683, 1967/1968.

[13] Józef Siciak. On some extremal functions and their applications in the theory of analytic functions of several complex variables. Trans. Amer. Math. Soc., 105:322-357, 1962.

[14] Józef Siciak. Extremal plurisubharmonic functions in $\mathbf{C}^{n}$. Ann. Polon. Math., 39:175-211, 1981.

[15] Henri Skoda. Morphismes surjectifs et fibrés linéaires semi-positifs. In Séminaire Pierre Lelong-Henri Skoda (Analyse), Année 1976/77, volume 694 of Lecture Notes in Math., pages 290-324. Springer, Berlin, 1978.

[16] Wilhelm Stoll. Value distribution on parabolic spaces. Lecture Notes in Mathematics, Vol. 600. Springer-Verlag, Berlin-New York, 1977.

[17] T. Winiarski. Approximation and interpolation of entire functions. Ann. Polon. Math., 23:259-273. (errata insert), 1970/1971.

[18] V. P. Zakharyuta. Extremal plurisubharmonic functions, orthogonal polynomials and Bernstein-Walsh theorem for analytic functions of several complex variables. Ann. Polon. Math., 33:137-148, 1974 (Russian).

[19] A. Zeriahi. Fonction de Green pluricomplexe à pôle à l'infini sur un espace de Stein parabolique et applications. Math. Scand., 69(1):89-126, 1991.

[20] A. Zeriahi. Approximation polynomiale et extension holomorphe avec croissance sur une variété algébrique. Ann. Polon. Math., 63(1):35-50, 1996.

[21] Ahmed Zeriahi. A criterion of algebraicity for Lelong classes and analytic sets. Acta Math., 184(1):113-143, 2000. 